\title{
Updated evolutionary sequences for hydrogen-deficient white dwarfs
}

\author{
María E. Camisassa, Leandro G. Althaus \\ Facultad de Ciencias Astronómicas y Geofísicas, Universidad Nacional de La Plata, Paseo \\ del Bosque s/n, 1900 La Plata, Argentina \\ Instituto de Astrofísica de La Plata, UNLP-CONICET, Paseo del Bosque s/n, 1900 La \\ Plata, Argentina \\ René D. Rohrmann \\ Instituto de Ciencias Astronómicas, de la Tierra y del Espacio (CONICET-UNSJ), Av. \\ España Sur 1512, J5402DSP, San Juan, Argentina \\ Enrique García-Berro, Santiago Torres \\ Departament de Física, Universitat Politècnica de Catalunya, c/Esteve Terrades 5, 08860 \\ Castelldefels, Spain \\ Institute for Space Studies of Catalonia, c/Gran Capità 2-4, Edif. Nexus 201, 08034 \\ Barcelona, Spain \\ and \\ Alejandro H. Córsico, Felipe C. Wachlin \\ Facultad de Ciencias Astronómicas y Geofísicas, Universidad Nacional de La Plata, Paseo \\ del Bosque s/n, 1900 La Plata, Argentina \\ Instituto de Astrofísica de La Plata, UNLP-CONICET, Paseo del Bosque s/n, 1900 La \\ Plata, Argentina
}

Received ; accepted 


\begin{abstract}
We present a set of full evolutionary sequences for white dwarfs with hydrogendeficient atmospheres. We take into account the evolutionary history of the progenitor stars, all the relevant energy sources involved in the cooling, element diffusion in the very outer layers, and outer boundary conditions provided by new and detailed non-gray white dwarf model atmospheres for pure helium composition. These model atmospheres are based on the most up-to-date physical inputs. Our calculations extend down to very low effective temperatures, of $\sim 2500 \mathrm{~K}$, provide a homogeneous set of evolutionary cooling tracks that are appropriate for mass and age determinations of old hydrogen-deficient white dwarfs, and represent a clear improvement over previous efforts, which were computed using gray atmospheres.
\end{abstract}

Subject headings: stars: evolution — stars: interiors — stars: white dwarfs 


\section{Introduction}

White dwarfs are the most common fossil stars within the stellar graveyard. In fact, it is well known that more than 90 per cent of all main sequence stars will finish their lives as white dwarfs. On their way to become fossil remnants, half or perhaps even more of the original mass of main sequence stars is recycled back to the interstellar medium, thus contributing to its enrichment in metals. Supported by electron degeneracy, white dwarfs continue to cool down for very long periods of time, allowing us to look back at early times. Moreover, their structural and evolutionary properties are now reasonably well understood - see for instance, the reviews of Fontaine \& Brassard (2008), Winget \& Kepler (2008) and Althaus et al. (2010b) - at least for moderately low luminosities. Consequently, the Galactic population of white dwarfs carries essential information about several fundamental issues (García-Berro \& Oswalt 2016) and is of crucial importance to study, among other interesting topics, the evolution of stars off the main sequence, the structure and evolution of our own Galaxy and of its components - including the thin and thick disks (Winget et al. 1987; García-Berro et al. 1988; García-Berro et al. 1999; Torres et al. 2002), the Galactic spheroid (Isern et al. 1998; García-Berro et al.|2004) and the system of open and globular clusters, see for instance García-Berro et al. (2010), Jeffery et al. (2011), Bono et al. (2013), Hansen et al. (2013) and Torres et al. (2015) for some examples - the behavior of matter at high densities and low temperatures (Garcia-Berro et al. 1988; Isern et al. 1991), and also the evolution of planetary systems across the several phases of stellar evolution (Farihi) 2016). Not only that, white dwarfs can be also used as astroparticle physics laboratories (Isern et al. 1992; Bischoff-Kim et al. 2008; Isern et al. 2008, 2010; Córsico et al. 2012), and to test a hypothetical secular variation of the fundamental constants (García-Berro et al. 1995; García-Berro et al. 2011; Berengut et al. 2013).

To undertake these tasks two conditions must be fulfilled. First, from the observational 
point of view we need observational data to which the theoretical models can be compared. Surveys such as the Sloan Digital Sky Survey (York et al. 2000) or the ESO Supernovae Type Ia Progenitor Survey (Napiwotzki et al. 2001), to put only two well-known examples, have provided us with an unprecedented wealth of information, allowing us to make meaningful comparisons of the theoretical cooling tracks with the observed degenerate sequences. Furthermore, it is foreseen that the results of future massive surveys, like the successive data releases of Gaia or the data obtained using the Large Synoptic Survey Telescope (Ivezic et al. 2008), will revolutionize the research field - see, for instance, Torres et al. (2005), and references therein. However, being this important, reliable cooling sequences aimed at reproducing the characteristics of the observed populations of white dwarfs are also needed. In particular, to use white dwarfs as reliable clocks to date stellar populations precise evolutionary models are required. This, in turn, means that all the relevant sources and sinks of energy must be carefully evaluated, and that a detailed and realistic treatment of the energy transport in their atmospheres is required. Also, ideally, a full end-to-end treatment of all the stellar evolutionary phases from the zero-age main sequence (ZAMS), through the red giant and the thermally pulsing phases to the planetary nebula and cooling stages would be needed. Only in this way state-of-the-art and realistic initial models for the cooling sequences can be obtained.

During the last decades, several new evolutionary models have been computed for white dwarfs with hydrogen-rich atmospheres (Wood 1992; Salaris et al. 1997; Hansen 1998, 1999; Salaris et al. 2000; Fontaine \& Brassard 2008; Salaris et al. 2010; Althaus et al. 2015; Camisassa et al. 2016). Nevertheless, about 20 per cent of all white dwarfs have helium-dominated atmospheres (the so-called class of non-DA white dwarfs). Most of these white dwarfs are thought to be the result of late thermal pulses experienced by post-asymptotic giant branch progenitors (Althaus et al. 2005). In contrast with the large efforts devoted to model the cooling of white dwarfs with hydrogen-rich atmospheres very 
few works have been devoted to study the cooling of white dwarfs with helium-dominated atmospheres. The most relevant studies are those of Wood (1995), Bergeron et al. (1995), Benvenuto \& Althaus (1999) and Althaus et al. (2009). However, most of these calculations rely on weak grounds. The reason for this is that the calculation of realistic cooling times for hydrogen-deficient white dwarfs strongly depends on the treatment of the energy transport through their atmospheres, particularly at low effective temperatures. The large densities that characterize helium white dwarf atmospheres, about $1 \mathrm{~g} / \mathrm{cm}^{3}$ in the coolest models, means that non-ideal effects have to be considered in the radiative calculations and in the equation of state. Thus, the modeling of such atmospheres becomes a tough endeavor, and clearly is a more complex task than computing cooling sequences for white dwarf with hydrogen-rich atmospheres.

This paper is precisely aimed at filling this gap. In particular, here we present a suite of cooling sequences for white dwarfs with pure helium atmospheres that supersede those sets of calculations previously mentioned. Our initial white dwarf models are the result of progenitor stars evolved self-consistently from the ZAMS through all the stellar evolutionary phases, including the thermally pulsing phase and the born again stage. The computation of progenitor evolution provides us with realistic chemical profiles at the beginning of the white dwarf phase. Our white dwarf sequences incorporate non-gray detailed atmospheres, and encompass a broad spectrum of masses.

Our paper is organized as follows. In Sect. 2, we briefly describe our numerical tools and the main ingredients of the evolutionary sequences, the model atmospheres and the initial models. In Sect. 3 we present the results of our white dwarf evolutionary sequences, in particular the cooling times, the role of carbon enrichment in the envelope, and the predicted observational appearance of our sequences. Finally, in Sect. 4 we summarize the main findings of the paper and we present our conclusions. 


\section{Numerical setup and input physics}

\subsection{Stellar code}

The evolutionary sequences presented in this study have been calculated using the LPCODE stellar evolutionary code - see Althaus et al. (2003), Althaus et al. (2005), Althaus et al. (2012), Althaus et al. (2015), and Miller Bertolami (2016) for relevant information about the the details of the code. LPCODE is a well-tested and calibrated code that has been widely used to study the formation and evolution of white dwarf stars - see Miller Bertolami et al. (2008), García-Berro et al. (2010), Althaus et al. (2010a), Renedo et al. (2010), Miller Bertolami et al. (2011), Wachlin et al. (2011), Córsico et al. (2012), Althaus et al. (2013), and references therein. More recently, the code has been used to generate a new grid of models for post-AGB stars (Miller Bertolami 2016). LPCODE has been tested against other evolutionary codes during the main sequence, red giant branch, and white dwarf regime (Salaris et al. 2013; Miller Bertolami 2016) with satisfactory results.

In what follows we describe the main input physics of the code that are relevant for the computation of the evolution of hydrogen-deficient white dwarfs. Convection is treated within the standard mixing length formulation, as given by the ML2 parameterization (Tassoul et al. 1990). The nuclear network for helium burning is identical to that described

in Althaus et al. (2005). In our sequences, some residual helium burning occurs at the very beginning of the cooling sequences. Radiative opacities are computed using the OPAL tables (Iglesias \& Rogers 1996). For the low-temperature regime, molecular opacities with varying carbon to oxygen ratios are used. To this end, we have adopted the low temperature opacities computed by Ferguson et al. (2005) and presented by Weiss \& Ferguson (2009). In our code, molecular opacities are computed by adopting the opacity tables with the correct abundances of the unenhanced metals (e.g. Fe) and carbon to oxygen ratio. Interpolation is carried out by means of separate quadratic interpolations in $R=\rho / T_{6}{ }^{3}$ and 
$T$, but linearly in $N_{\mathrm{C}} / N_{\mathrm{O}}$. Conductive opacities are computed adopting the treatment of Cassisi et al. (2007). We consider the equation of state of Magni \& Mazzitelli (1979) for the low-density regime, while for the high-density regime, we employ the equation of state of

Segretain et al. (1994) which accounts for all the important contributions for both the solid and liquid phases. Neutrino emission rates for pair, photo, and bremsstrahlung processes are those of Itoh et al. (1996), while for plasma processes we follow the treatment presented in Haft et al. (1994).

As the white dwarf cools down we take into account abundance changes resulting from convective mixing and from element diffusion due to gravitational settling, chemical and thermal diffusion of ${ }^{4} \mathrm{He},{ }^{12} \mathrm{C},{ }^{13} \mathrm{C},{ }^{14} \mathrm{~N}$ and ${ }^{16} \mathrm{O}$ - see Althaus et al. (2003) for details. During the white dwarf regime and for effective temperatures smaller than $50000 \mathrm{~K}$, outer boundary conditions are derived from non-gray model atmospheres for pure helium composition - see Sect. 2.2. Energy sources resulting from crystallization, as the release of latent heat and of gravitational energy associated to carbon and oxygen phase separation upon crystallization are also taken into account. These energy sources are considered self-consistently and locally coupled to the full set of equations of stellar evolution. In particular, our treatment of crystallization includes the use of the most up-to-date phase diagram (Horowitz et al. 2010) of dense carbon-oxygen mixtures appropriate for white dwarf interiors, which is based on direct molecular dynamics simulations - see Althaus et al. (2012) for the implementation of this treatment in LPCODE.

\subsection{Stellar atmospheres}

Usually, for hydrogen-deficient white dwarfs the surface boundary conditions needed to integrate the equations of stellar evolution are taken from the gray atmosphere approximation. However, in the late stages of white dwarf cooling precise boundary 


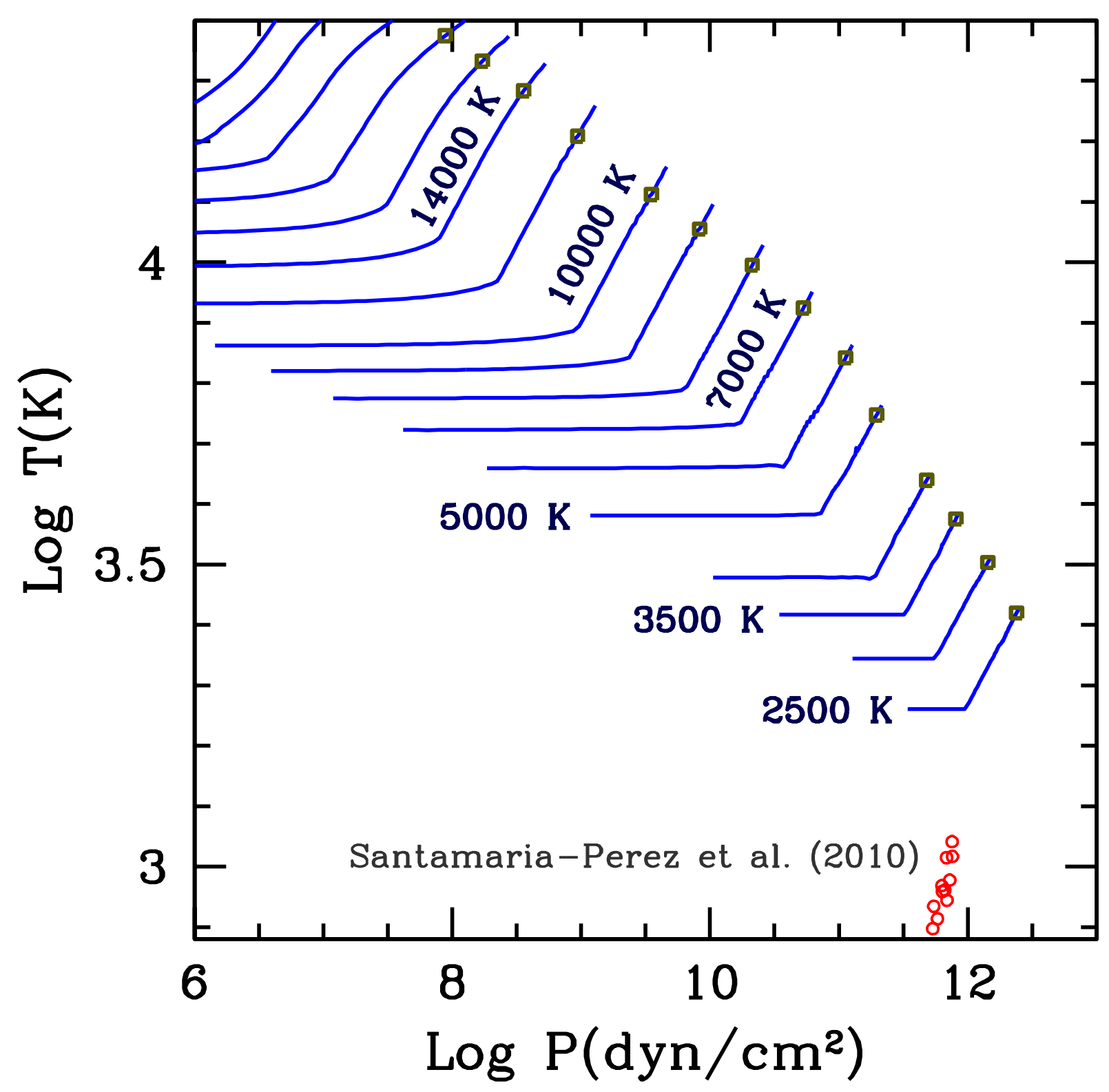

Fig. 1.- Temperature and pressure stratifications at $10^{-6} \leq \tau_{\text {Ross }} \leq 100$ for helium atmospheres with $\log g=8$ and various effective temperatures (some of them indicated on the plot). Squares display the optical depth $\tau_{\text {Ross }}=25.1189$ where the atmospheres are attached to interior models. Circles show the melting data on helium reported by Santamaría-Pérez et al. (2010). 
conditions are required because convective coupling between the envelope and the degenerate core occurs at low effective temperatures. In contrast with nearly all the calculations available so far, the outer boundary conditions employed in this work are obtained from detailed non-gray model atmospheres for $T_{\text {eff }} \leq 50000 \mathrm{~K}$. The location of this boundary was taken at a large optical depth (specifically, at the Rosseland mean optical depth $\left.\tau_{\text {Ross }}=25.1189\right)$ where the diffusion approximation, limiting form of the transfer equation used by the stellar code, is valid — see Rohrmann et al. (2012).

Model atmospheres were computed assuming constant gravity, hydrostatic equilibrium, local thermodynamic equilibrium, and energy flux conservation. The numerical code is a new and improved version of a previously existing code developed by Rohrmann et al. (2002), appropriately modified to take into account the high density effects expected in cool helium atmospheres. In the new code, the equations of monochromatic radiative transfer and the constant flux condition, including convective and conductive energy fluxes, are solved following a Rybicki scheme (Gustafsson \& Nissen 1972). Convective transport is treated within the ML2 version of the mixing-length theory, and the conductive opacity data is taken from Cassisi et al. (2007). The equation of state used in the atmospheric code is the He.REOS.3 model from Becker et al. (2014), supplemented with a chemical model based on the occupation probability formalism to solve the ionization equilibrium for He, $\mathrm{He}^{+}, \mathrm{He}^{++}, \mathrm{e}^{-}, \mathrm{He}^{-}$, and $\mathrm{He}_{2}^{+}$- see Hummer \& Mihalas (1988) and also Rohrmann et al. (2002). The radiative opacity sources included in the code comprise bound-bound (He, $\left.\mathrm{He}^{+}\right)$, bound-free $\left(\mathrm{He}, \mathrm{He}^{+}, \mathrm{He}^{2+}\right)$ and free-free $\left(\mathrm{He}, \mathrm{He}^{+}, \mathrm{He}^{2+}, \mathrm{He}^{-}\right)$processes, electron and Rayleigh scattering, and collision-induced absorption by atoms (Kowalski 2014). The density effects on the opacity described in Iglesias et al. (2002) were omitted in our code, because unfortunately such study does not cover a broad range of temperatures and densities as required in the present calculations. However, we have estimated the impact of these high density effects on the evolutionary timescales, and we found that the differences 


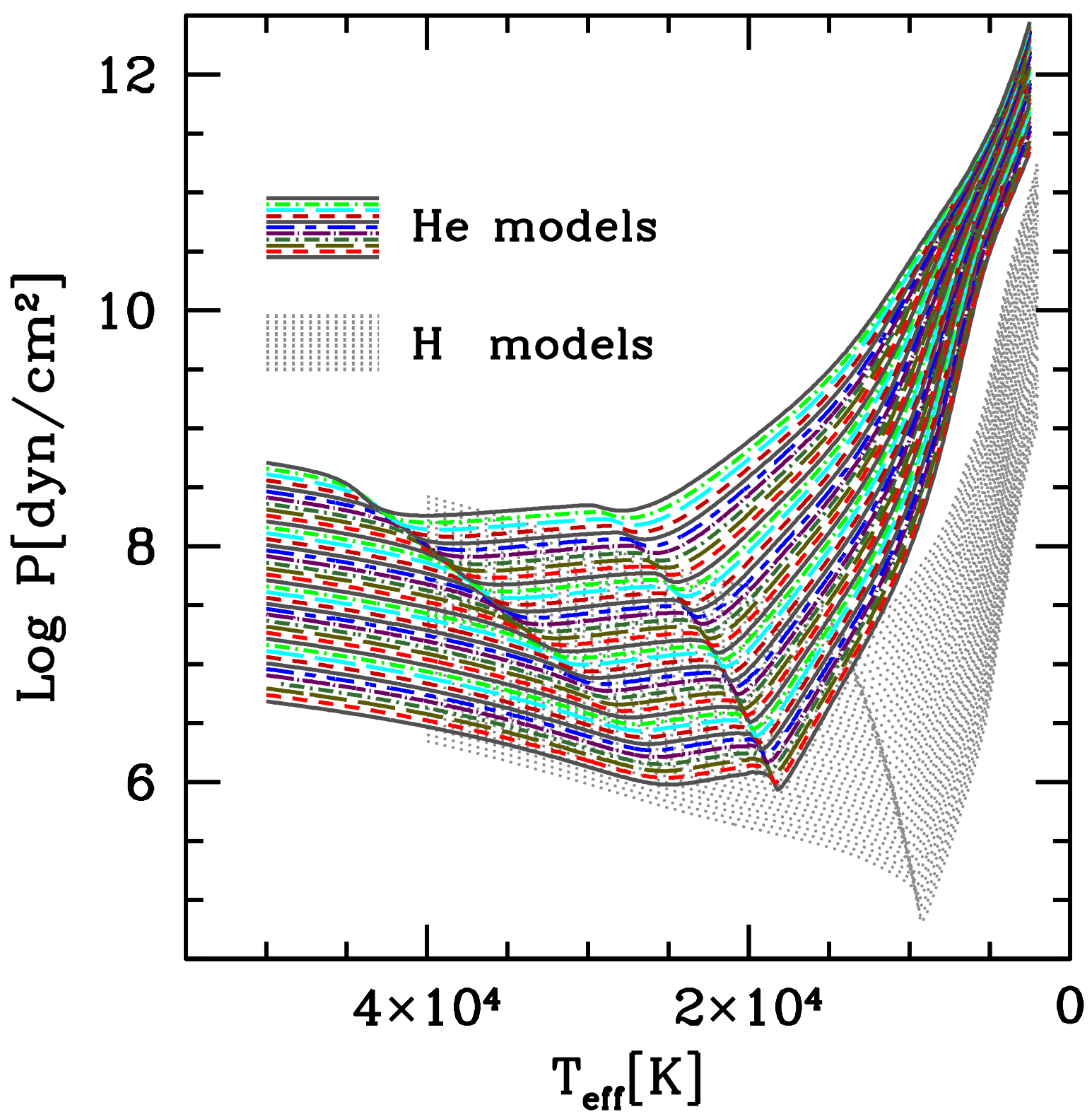

Fig. 2.- Pressure at $\tau_{\text {Ross }}=25.1189$ as a function of the effective temperature obtained for pure helium (solid and dashed lines) and pure hydrogen (dotted lines) atmospheres at $5.5 \leq \log g \leq 9.5$ (lines from bottom to top). 
in the cooling times would be less than $2 \%$.

Fig. 1 displays the temperature-pressure profiles of helium atmospheres for models calculated at $\log g=8$ and different effective temperatures. Temperature profiles are essentially isothermal in the outer and transparent layers $\left(\tau_{\text {Ross }} \lesssim 10^{-4}\right.$ for the coolest models), while convection becomes very efficient and the dominant mechanism of energy transport at the bottom layers $\left(\tau_{\text {Ross }} \geq 1\right)$ of cool atmospheres $\left(T_{\text {eff }} \lesssim 20000 \mathrm{~K}\right)$. Fig. 1 also shows the melting points reported by Santamaría-Pérez et al. (2010), which correspond to the liquid to solid phase transition of pure helium. These results show that hydrogendeficient white dwarfs evolve towards complex physical conditions in their atmospheres as they cool down.

The grid of atmosphere models calculated to provide outer boundary conditions to the evolutionary calculations covers a range of effective temperatures from $50000 \mathrm{~K}$ to $2500 \mathrm{~K}$ in steps of $100 \mathrm{~K}$, and surface gravities within $5.5 \leq \log g \leq 9.5$ in steps of 0.1 dex. Pressures and temperatures (at $\left.\tau_{\text {Ross }}=25.1189\right)$ required to integrate the stellar structure equations are shown in Figs. 2 and 3 , respectively. Solid and dashed lines correspond to our pure-helium model atmospheres, while dotted lines represent data from pure hydrogen models (Rohrmann et al. 2012). The pressures in helium and hydrogen atmospheres differ markedly at low effective temperatures, as a consequence of the differences in the opacity. The ripples seen around $T_{\text {eff }} \approx 40000 \mathrm{~K}$ and $20000 \mathrm{~K}$ in helium models $(10000 \mathrm{~K}$ in hydrogen ones) in Figs. 2 and 3 , are a consequence of $\mathrm{He}^{2+}$ and $\mathrm{He}^{+}\left(\mathrm{H}^{+}\right)$recombinations.

\subsection{Initial models}

The initial models for our evolving hydrogen-deficient white dwarf sequences were derived from the full evolutionary calculations of their progenitor stars for $Z=0.02$ 


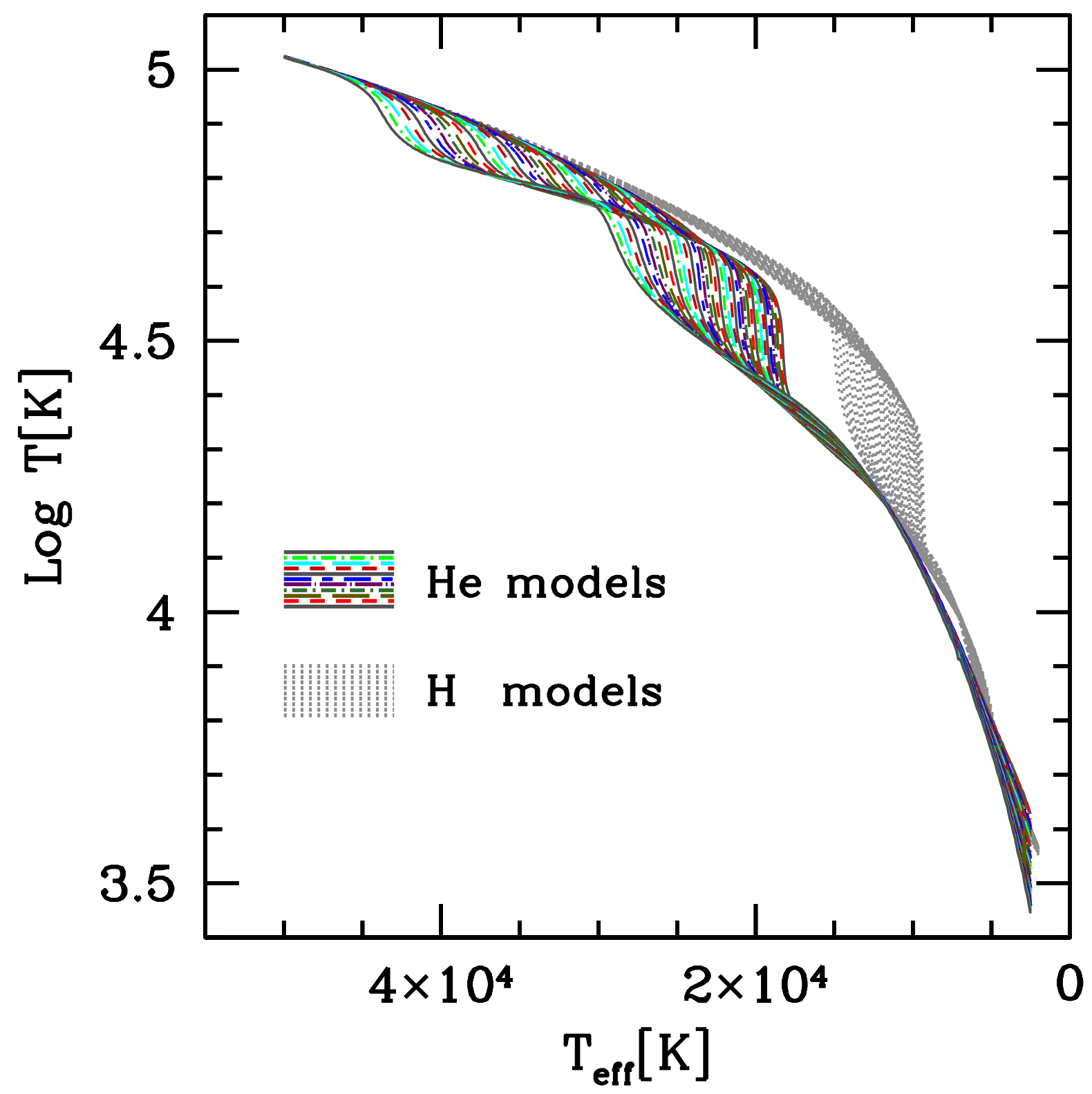

Fig. 3.- Temperature at $\tau_{\text {Ross }}=25.1189$ as a function of the effective temperature obtained for pure helium (solid and dashed lines) and pure hydrogen (dotted lines) atmospheres at $5.5 \leq \log g \leq 9.5$ (lines from top to bottom). 
(Miller Bertolami \& Althaus 2006). The evolution of the white dwarf progenitors was computed from the ZAMS through the thermally-pulsing and mass-loss phases on the asymptotic giant branch (AGB), and finally to the born-again stage where the remaining hydrogen is violently burned. After the born-again episode, the hydrogen-deficient, quiescent remnants of helium burning evolve at constant luminosity with a surface chemical composition rich in helium, carbon and oxygen, typical of PG 1159 stars (Miller Bertolami \& Althaus 2006). As it will be shown below, such a detailed treatment of the evolutionary history of progenitors stars with different initial stellar masses and distinct chemical compositions is a key ingredient in computing accurate cooling sequences for cool hydrogen-deficient white dwarfs. The initial masses of our sequences and the white dwarf masses resulting from the born again episode are listed in Table 1, In particular, in this table we list, from left to right, the white dwarf mass, the initial mass and the total mass of helium of the envelope (all of them in solar units), and the effective temperature, the surface luminosity, and the surface gravity at the point of maximum effective temperature. Finally, also listed are the initial surface abundances (by mass) of helium, carbon and oxygen. For the $1.0 M_{\odot}$ initial model, two different sequences were computed with two different mass-loss rates during the AGB evolution. Thus, we obtain a different number of thermal pulses and, in the end, two different remnant masses of 0.515 and $0.542 M_{\odot}$. Finally, in order to cover a wide range of stellar masses, we generated an additional $1.0 M_{\odot}$ white dwarf model by artificially scaling our $0.87 M_{\odot}$ white dwarf model. Hence, the masses of our white dwarf sequences range from 0.51 to $1.0 M_{\odot}$. This mass interval covers most of the observed stellar mass range of hydrogen-deficient white dwarfs. Note that for these sequences, the expected range of helium envelope masses spans more than one order of magnitude. In this work, emphasis is placed on the late stages of white dwarf evolution, where the impact of detailed model atmosphere is larger. The evolutionary stages covering the hot pre-white dwarf phases — namely, the PG 1159 regime and the following ones — were studied in 
detail in Althaus et al. (2009). Consequently, we will not pay much attention to the very early evolutionary phases. Instead, the evolution of these model stars has been followed until very low surface luminosities, $\log \left(L / L_{\odot}\right)=-5.0$, and will be discussed in depth here.

The initial chemical abundance distribution (that is, at the beginning of the cooling track) for some of our selected white dwarf models is discussed with the help of Figs. 4 and 5, which display the abundance by mass of ${ }^{4} \mathrm{He},{ }^{12} \mathrm{C},{ }^{13} \mathrm{C},{ }^{14} \mathrm{~N}$, and ${ }^{16} \mathrm{O}$ in terms of the outer mass fraction. In particular, the upper panels of these figures show the abundances at the beginning of the cooling track of the sequences with 0.515 and $0.870 M_{\odot}$, respectively. For illustrative purposes, the inner chemical structure expected for hydrogen-rich white dwarf structures of similar stellar mass is shown in the bottom panel of each figure. Note the dependence of the chemical abundance profile on the stellar mass. The signatures left by the born again episode in the outer layer chemical stratification is clearly visible. In particular, it can be seen that hydrogen is almost completely burned in these layers. In passing we note that because we are interested in the evolution of hydrogen-deficient white dwarfs, we have artificially removed all the traces of hydrogen in our initial models. In particular, note the high amount of carbon and nitrogen expected in hydrogen-deficient white dwarfs, see Miller Bertolami \& Althaus (2006) for details. This will be a key issue to be considered when describing the final phases of cooling of these white dwarfs. Note in particular that ${ }^{13} \mathrm{C}$ reaches abundances as high as 0.05 by mass throughout the envelope. It is clear that, in order to obtain realistic initial chemical profiles for hydrogen-deficient white dwarfs, the progenitor evolution through the born-again scenario must be taken into account. Specifically, the resulting chemical profiles in the outer layers differ markedly from those predicted by progenitors that produce hydrogen-rich white dwarfs. 

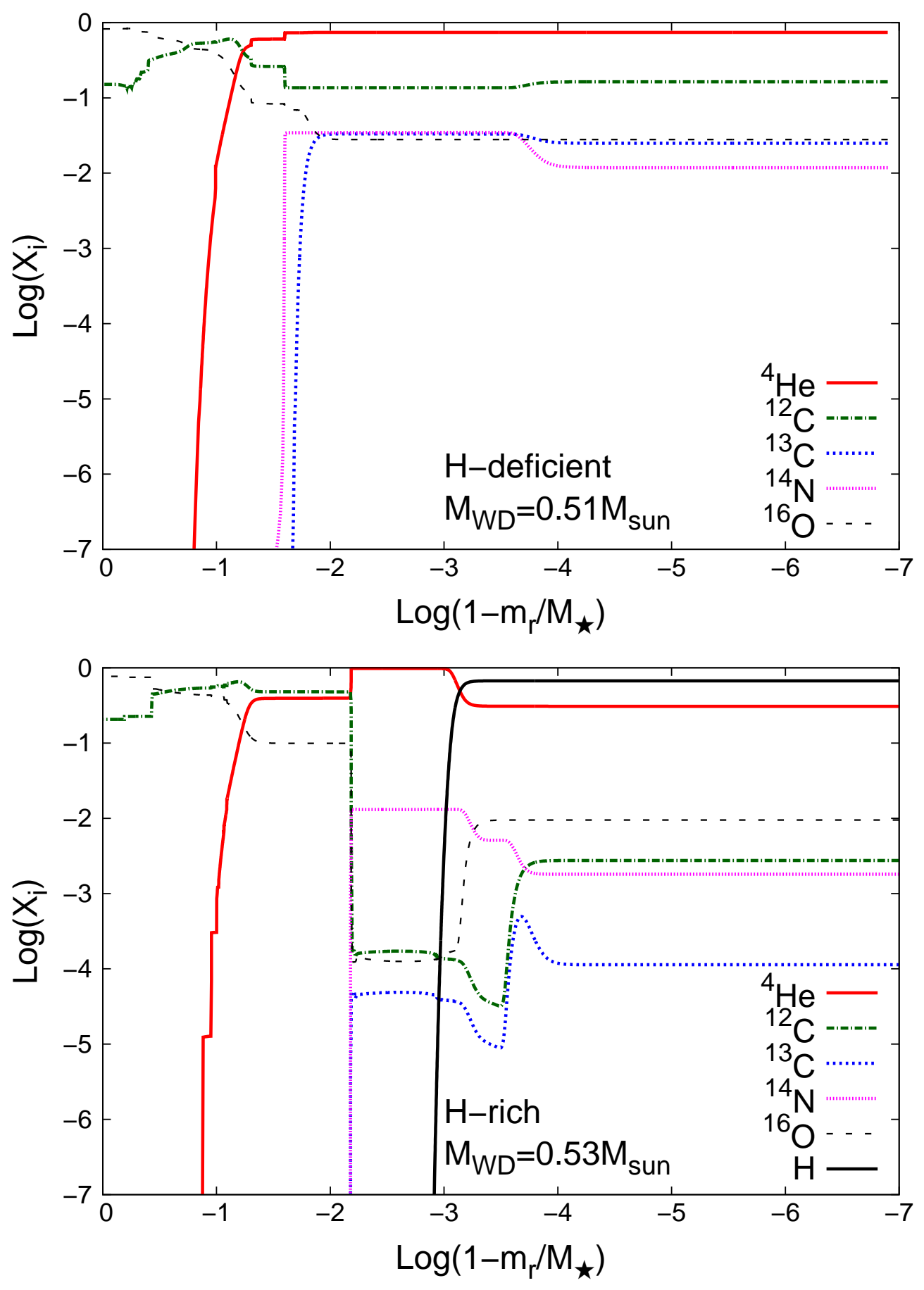

Fig. 4.- Upper panel: initial chemical profiles for our $0.51 M_{\odot}$ hydrogen-deficient white dwarf model in terms of the outer mass fraction. Bottom panel: initial chemical profiles for a $0.53 M_{\odot}$ hydrogen-rich white dwarf model (Camisassa et al. 2016) 

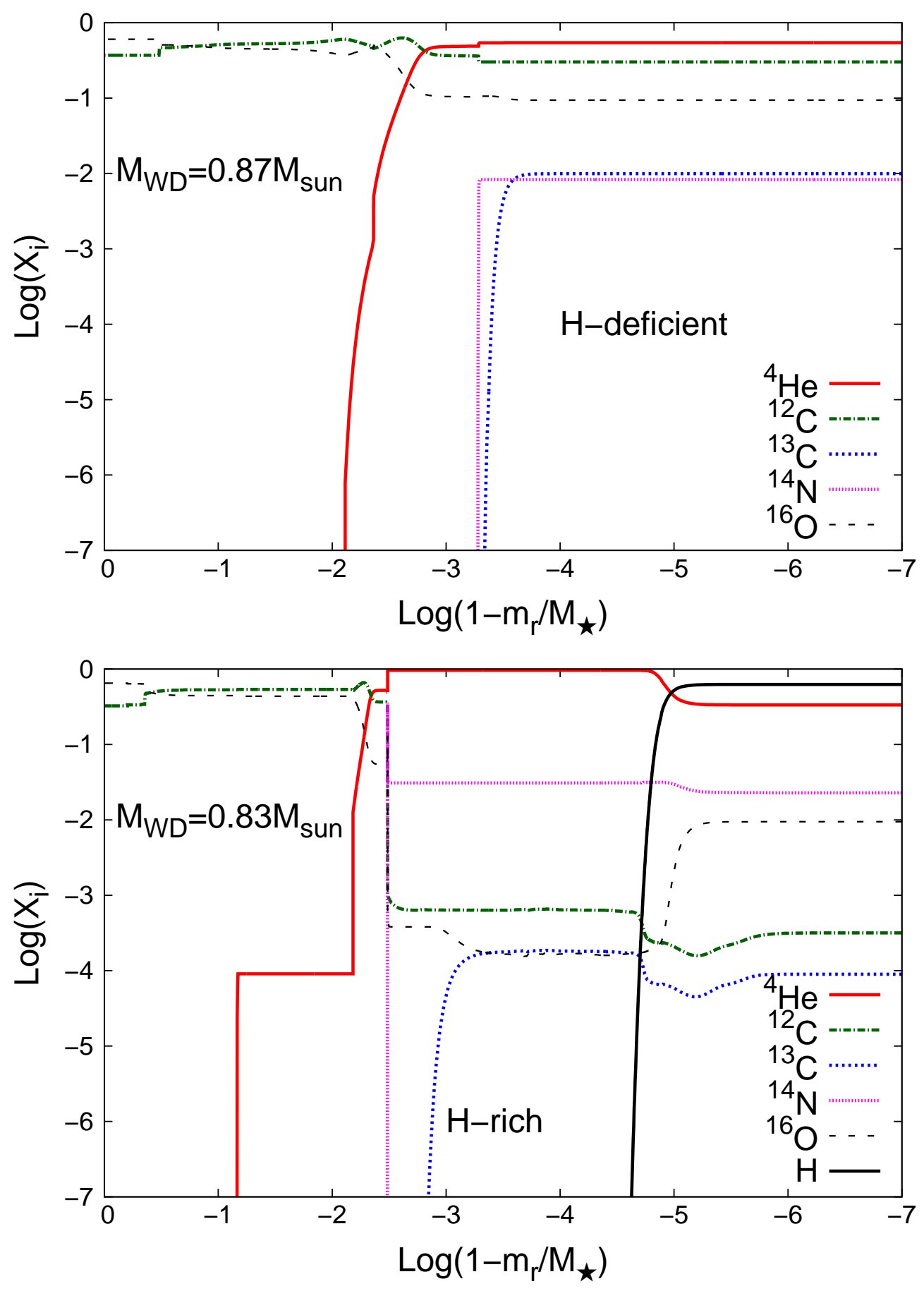

Fig. 5.- Upper panel: initial chemical profiles for our $0.87 M_{\odot}$ hydrogen-deficient white dwarf model in terms of the outer mass fraction. Bottom panel: initial chemical profiles for a $0.83 M_{\odot}$ hydrogen-rich white dwarf model (Camisassa et al. 2016) 


\section{Results}

A global view of the main phases of the evolution of a typical hydrogen-deficient white dwarf is shown in Fig. 6. In this figure the different luminosity contributions are plotted for our $0.58 M_{\odot}$ white dwarf sequence resulting from a progenitor star of $2.5 M_{\odot}$. During the entire white dwarf evolution, the release of gravothermal energy is the dominant energy source. Also, during the very early stages of the white dwarf cooling phase, helium burning contributes somewhat to the white dwarf energy budget. However, shortly after it becomes negligible. At $\log (t) \sim 5.5$ neutrino emission becomes an important energy sink, even larger than the star luminosity. At that time, gravitational settling has already depleted all the heavy elements from the surface, leaving an atmosphere made of almost pure helium. However, gravitational settling is still acting in the envelope, and chemical and thermal diffusion are still smoothing the inner chemical interfaces. The resulting chemical stratification will be discussed below. From $\log (t) \sim 6$ to 7.1, the energy lost by neutrino emission is of about the same order of magnitude as the gravothermal energy release. As the white dwarf cools, neutrino emission ceases and, consequently, the neutrino luminosity abruptly drops. During this phase, the outer convective zone grows inwards and at $\log (t) \sim 8.2$, it penetrates into layers where heavy elements as carbon and oxygen are abundant. Consequently, convective mixing dredges up these heavy elements, and the surface composition changes. In particular, the outer layers are predominantly enriched in carbon. This dredge-up episode alters markedly the evolutionary timescales of the white dwarf, as it will be demonstrated below. Finally, at $\log (t) \sim 9$ crystallization sets in at the center of the white dwarf. This results in the release of latent heat and gravitational energy due to carbon-oxygen phase separation. Note that, as a consequence of this energy release, during the crystallization phase, the surface luminosity is larger than the gravothermal luminosity. This phase lasts for $3.2 \times 10^{9}$ years. It is also important to note that during the crystallization phase, carbon is still being dredged-up to the surface. Finally, at 
$\log (t) \sim 9.9$, the temperature of the crystallized core drops below the Debye temperature, and consequently, the heat capacity decreases. Thus, the white dwarf enters into the so-called "Debye cooling phase", where the luminosity drops.

The behavior of the cooling sequences during the late stages can be understood by examining Fig. 7, which illustrates the run of the central temperature in terms of the surface luminosity for our $0.58 M_{\odot}$ hydrogen-deficient white dwarf model. In the interests of comparison, we also include in this figure the predictions for a hydrogen-rich white dwarf model of similar mass. The thick segments in the cooling curves indicate the crystallization phase. The shaded areas mark the occurrence of convective coupling in each sequence, that is, when the outer convective zone reaches the degenerate core, and thus the energy transport through the outer layers becomes more efficient (Fontaine et al. 2001). For our hydrogen-deficient white dwarf model, crystallization sets in at $\log \left(L / L_{\odot}\right) \sim-3.4$, whereas in the hydrogen-rich model crystallization begins when $\log \left(L / L_{\odot}\right) \sim-3.9$. Crystallization sets in at higher luminosities in hydrogen-deficient white dwarf models, and this is because convective coupling occurs at higher luminosities in these white dwarfs. In fact, convective coupling causes the change of slope in the cooling curves, and the core temperature strongly decreases. This result, which is independent of the white dwarf mass, leads to marked differences in the evolution of both types of white dwarfs.

\subsection{Chemical evolution}

The chemical profiles at selected evolutionary stages during the white dwarf cooling phase are shown in Fig. 8 for our $0.58 M_{\odot}$ white dwarf model. Each panel is labeled with the logarithm of the luminosity (in solar units) and the logarithm of the effective temperature of the evolutionary model. We show the logarithm of the abundances of ${ }^{4} \mathrm{He},{ }^{12} \mathrm{C},{ }^{13} \mathrm{C},{ }^{14} \mathrm{~N}$ and ${ }^{16} \mathrm{O}$ in terms of the outer mass fraction. The upper panel corresponds to the beginning of 


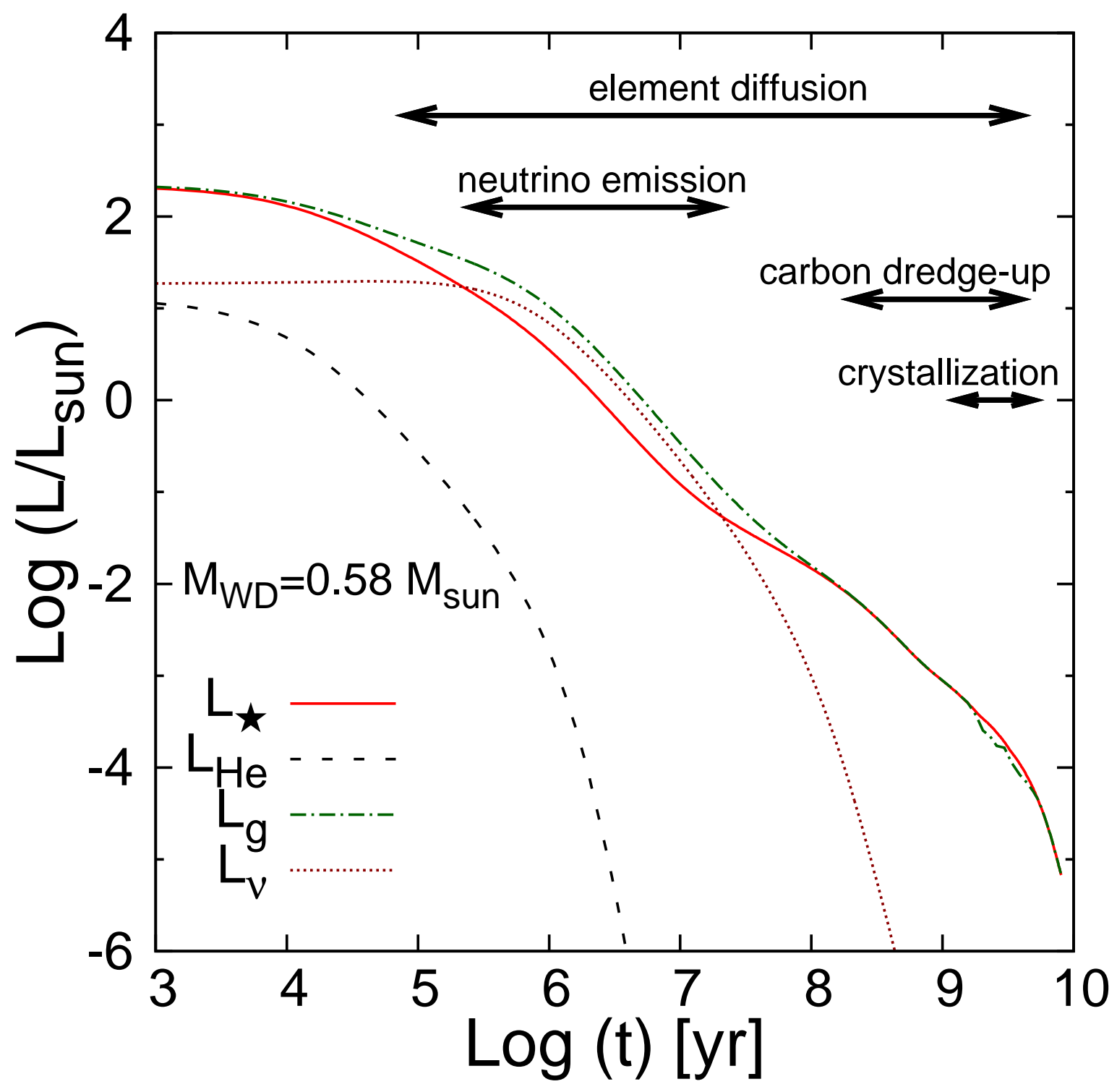

Fig. 6. - Time dependence of the different luminosity contributions for our $0.58 M_{\odot}$ hydrogen-deficient white dwarf sequence. We show the surface luminosity, $L_{\star}$ (solid line), the luminosity due to helium-burning, $L_{\mathrm{He}}$ (dashed line), the rate of gravothermal (compression plus thermal) energy release, $L_{\mathrm{g}}$ (dot-dashed line), and the neutrino losses, $L_{\nu}$ (dotted line). Time is expressed in years since the moment when the star reaches the maximum effective temperature. The various physical processes occurring as the white dwarf cools down are also indicated in the figure. 


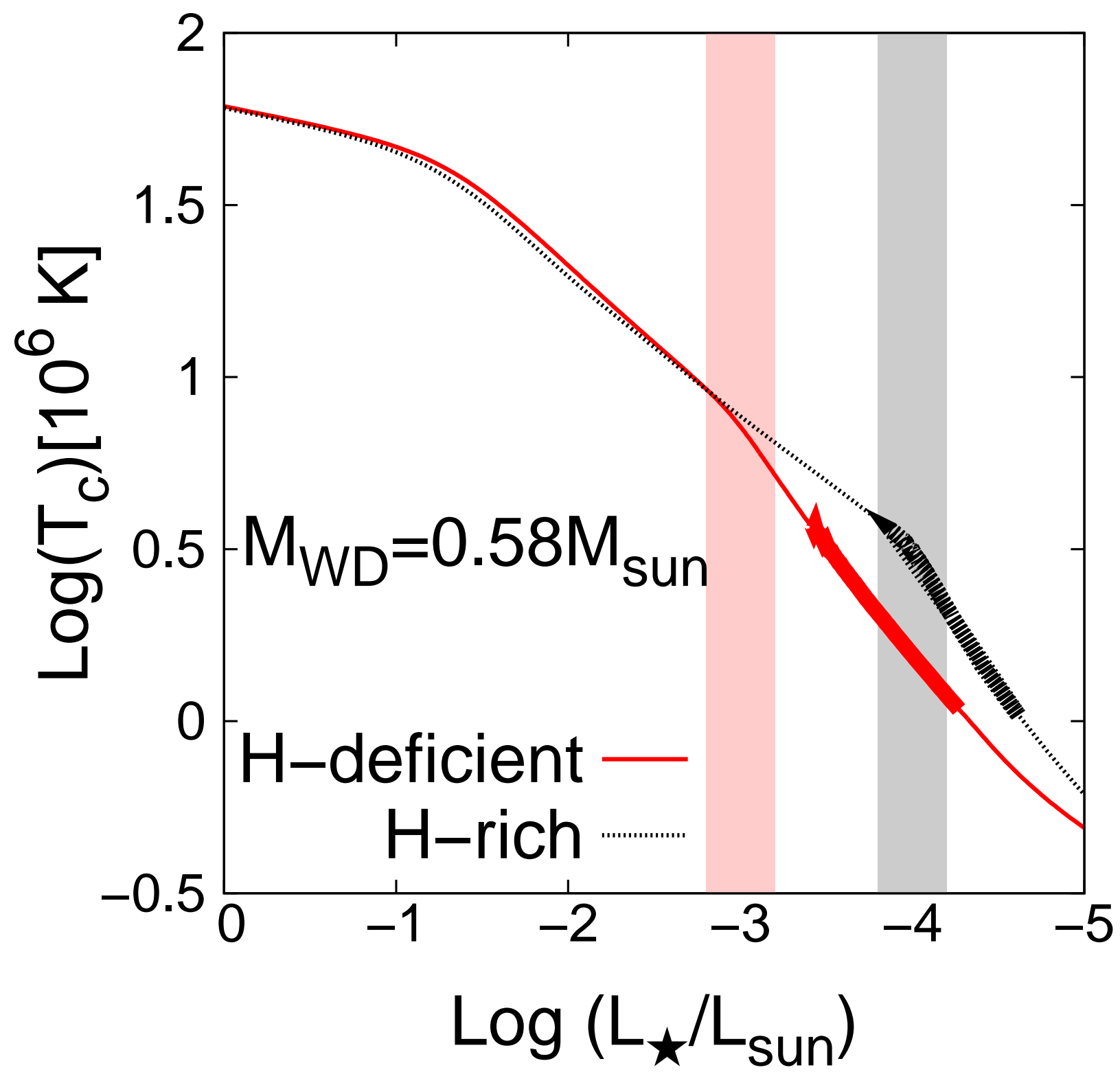

Fig. 7.- Central temperature in terms of the star luminosity for our $0.58 M_{\odot}$ hydrogendeficient white dwarf model (solid red line), together with a $0.58 M_{\odot}$ hydrogen-rich model from Camisassa et al. (2016) (dotted black line). Thick segments indicate the core crystallization phase. Shaded areas indicate the occurrence of convective coupling. 


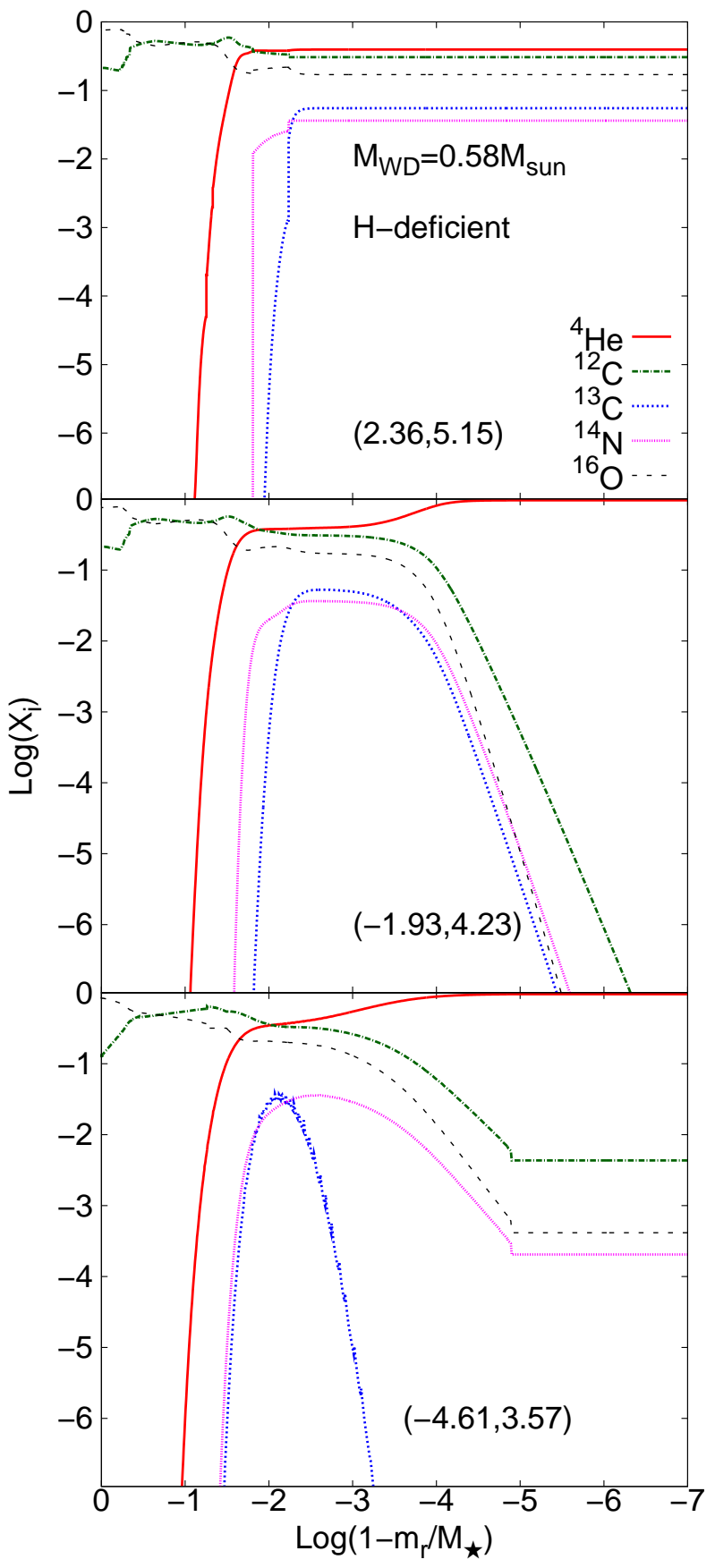

Fig. 8.- Abundance by mass of ${ }^{4} \mathrm{He},{ }^{12} \mathrm{C},{ }^{13} \mathrm{C},{ }^{14} \mathrm{~N}$ and ${ }^{16} \mathrm{O}$ as a function of the outer mass fraction, $\log \left(1-m_{r} / M_{\star}\right)$, for our $0.58 M_{\odot}$ white dwarf model at three selected evolutionary stages. The logarithm of the luminosity (in solar units) and the logarithm of the effective temperature are indicated in brackets in each panel. 
the white dwarf phase. That is, we show the chemical profiles at the moment of maximum effective temperature. As mentioned, these profiles are the result of the entire previous evolution of the progenitor star, particularly of the born again episode. After $1.26 \times 10^{8}$ years, the white dwarf has cooled down to $17000 \mathrm{~K}$ (middle panel), and the chemical composition displays the effects of the action of diffusion processes. Gravitational settling has depleted all heavy elements from the outer layers, leaving an envelope composed by pure ${ }^{4}$ He. Chemical and thermal diffusion have already smoothed the chemical interfaces. At this point, the outer convective zone is growing and penetrating into deeper layers, but has not yet reached the regions where carbon is abundant. Shortly after, when $T_{\text {eff }} \sim 15000 \mathrm{~K}$, the outer convective zone reaches the tail of the carbon distribution, and convection dredges-up carbon to the surface layers, increasing the carbon surface abundance. Carbon dredge-up by convection will continue for $4 \times 10^{9} \mathrm{yr}$, until $T_{\text {eff }} \sim 5000 \mathrm{~K}$. During this period, thermal and chemical diffusion are still acting and further smooth the chemical interfaces, thus increasing the amount of carbon that is finally dredged-up to the surface. It is important to remark that convection also dredges-up other chemical species, but carbon is the one that is primarily dredged-up. Also, during this period, crystallization starts in the degenerate core, changing as well the carbon and oxygen chemical profiles in the central regions of the star. The final chemical abundances, at $T_{\text {eff }} \sim 3700 \mathrm{~K}$ are shown in the bottom panel of Fig. 8 . Note that at this point, convection has dredged-up a large amount of carbon to the surface.

For a better understanding of the carbon dredge-up process, in Fig. 9 we show using a red solid line the location of the base of the outer convective zone of our $0.51 M_{\odot}$ white dwarf sequence in terms of the effective temperature. For comparison, we have also included using a black solid line the predictions for a $0.51 M_{\odot}$ hydrogen-deficient white dwarf sequence resulting from considering the outer boundary conditions of an Eddington atmosphere model. As the white dwarf cools, the outer convective zone penetrates into deeper regions. At low effective temperatures, convection eventually reaches deeper layers 


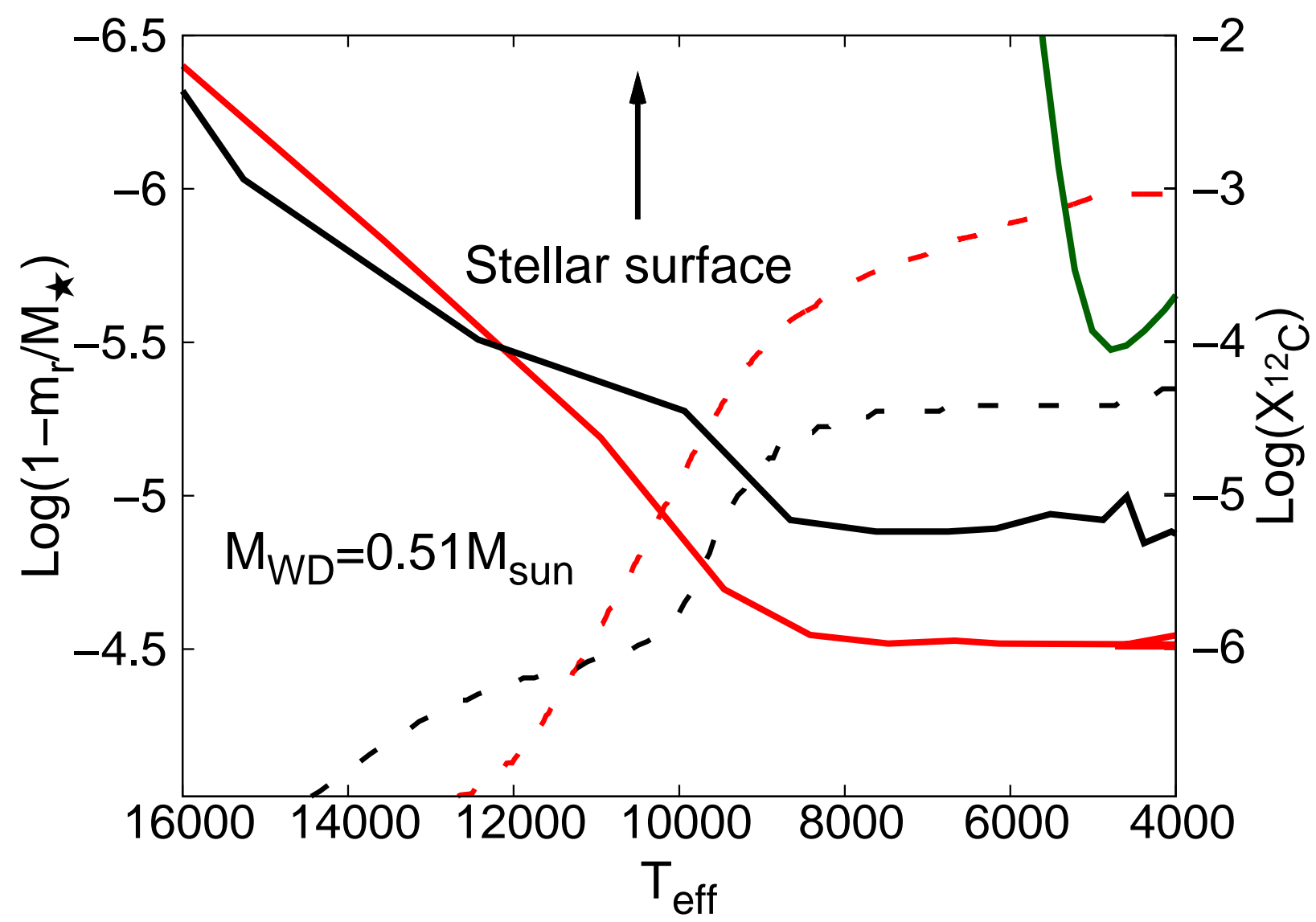

Fig. 9.- Effect of convection on the carbon abundance in the envelope as a function of effective temperature for our $0.51 M_{\odot}$ white dwarf model. The red and black solid lines indicate, respectively, the base of the outer convective zone resulting from considering a detailed model atmosphere and the Eddington model atmosphere as outer boundary conditions. The red and black dashed lines show the surface carbon abundances resulting from considering the detailed and the Eddington model atmosphere, respectively. The green solid line indicates the base of the outer convective zone in a hydrogen-rich white dwarf model of similar mass. The deeper convection zone of the detailed model atmosphere results in more efficient dredge-up of carbon and a higher carbon abundance at the surface. 
in the case that a detailed treatment of the atmosphere is considered, thus yielding larger final surface carbon abundances (dashed red line), as compared with the situation in which the Eddington atmosphere is considered (dashed black line). Note also that for a hydrogen-deficient white dwarf the outer convective zone penetrates much deeper in the star than in the case in which a hydrogen-rich white dwarf is considered.

All our white dwarf models experience carbon dredge-up due to convective mixing. This is illustrated in Fig. 10, where the surface abundance of carbon of our sequences is displayed in terms of the effective temperature. All the white dwarf cooling sequences exhibit roughly the same behavior. As a result of gravitational settling, surface carbon abundance is negligible until $\log \left(T_{\text {eff }}\right) \sim 4.1$. At this point, the outer convective zone penetrates into the carbon-rich layers, and consequently the surface carbon abundance increases. This process is also favored by the occurrence of thermal and chemical diffusion in deeper layers. When $\log \left(T_{\text {eff }}\right) \sim 3.7$, the penetration of the outer convective zone ceases, and the surface abundance of carbon remains essentially constant. The final surface carbon abundance depends on the stellar mass, as well as on the initial chemical abundances. The average final surface carbon abundance of our models is about $5 \times 10^{-3}$. This value is high enough to strongly modify the evolutionary timescales of hydrogen-deficient white dwarfs, as it will be shown below. For the sake of comparison, we also performed some additional calculations considering the Eddington atmospheres as outer boundary conditions, and we found that in this case the resulting evolutionary sequences also experience appreciable carbon enrichment in the envelope as a result of convective mixing, although the carbon enhancements in the very outer layers of the star are substantially smaller. Our results can explain the existence of DQ white dwarfs, which exhibit traces of carbon in their atmospheres below $\sim 10000 \mathrm{~K}$. The white dwarf models presented in this work undergo a spectral type transition from DB type to DQ type as their atmospheres become carbon enriched. The filled circles in Fig. 10 represent the DQ white dwarfs studied by Koester \& Knist (2006). 
Table 1: Main characteristics of our initial white dwarf models.

\begin{tabular}{ccccccccc}
\hline \hline$M_{\mathrm{WD}}$ & $M_{\mathrm{ZAMS}}$ & $M_{\mathrm{He}}$ & $\log T_{\mathrm{eff}}[\mathrm{K}]$ & $\log \left(L / \mathrm{L}_{\odot}\right)$ & $\log g\left[\mathrm{~cm} \mathrm{~s}^{-2}\right]$ & $X_{\mathrm{He}}$ & $X_{\mathrm{C}}$ & $X_{\mathrm{O}}$ \\
\hline 0.515 & 1.00 & 0.0219 & 5.0634 & 2.6868 & 6.6713 & 0.7437 & 0.1637 & 0.0279 \\
0.542 & 1.00 & 0.0072 & 5.1650 & 3.0546 & 6.7412 & 0.2805 & 0.4064 & 0.2127 \\
0.584 & 2.50 & 0.0060 & 5.2398 & 3.2574 & 6.8615 & 0.3947 & 0.3060 & 0.1704 \\
0.664 & 3.50 & 0.0036 & 5.3578 & 3.3611 & 7.0813 & 0.4707 & 0.3260 & 0.1234 \\
0.741 & 3.75 & 0.0019 & 5.4535 & 3.8069 & 7.2701 & 0.4795 & 0.3361 & 0.1390 \\
0.870 & 5.50 & 0.0009 & 5.5829 & 4.0961 & 7.5679 & 0.5433 & 0.3012 & 0.0938 \\
\hline \hline
\end{tabular}

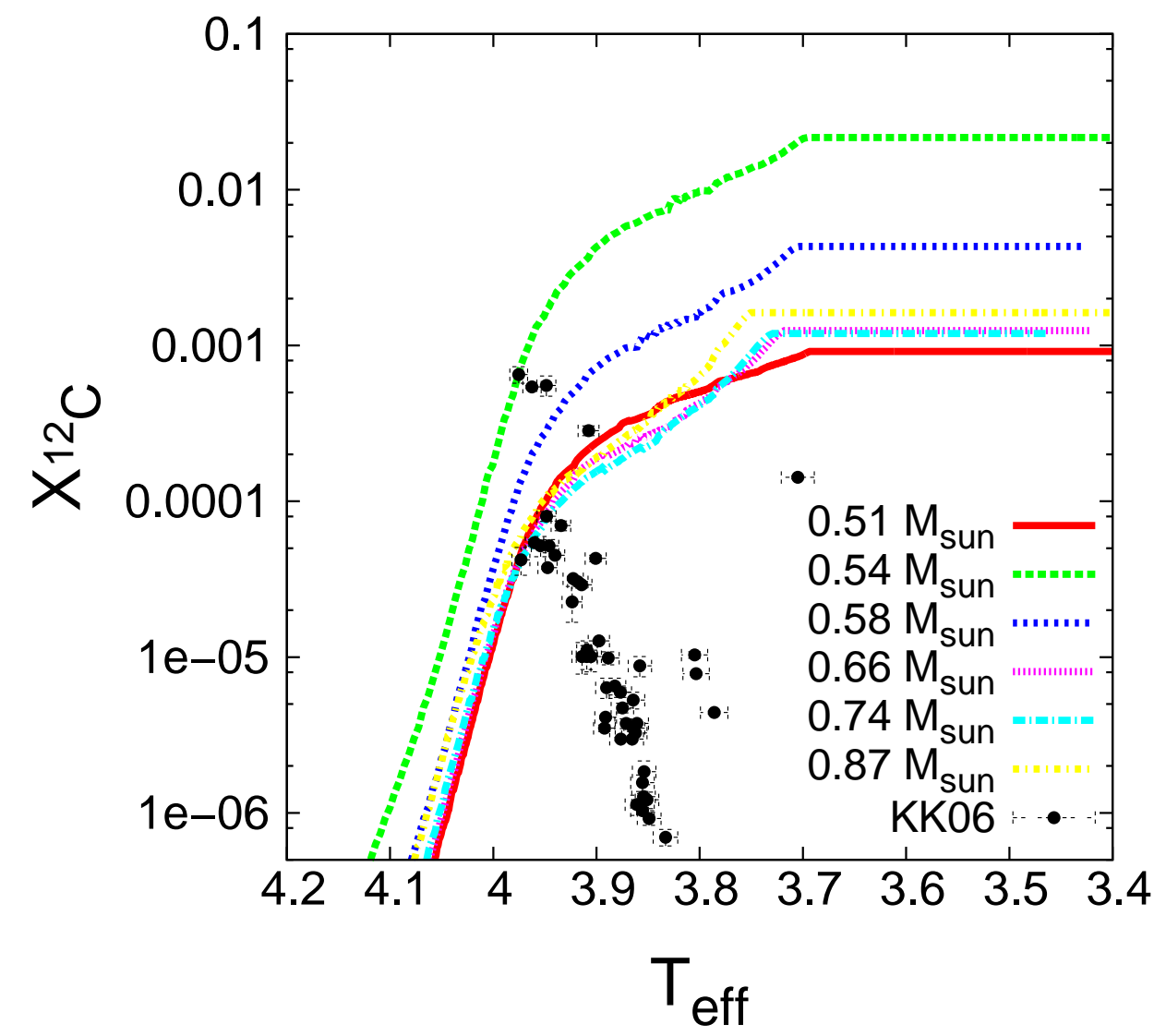

Fig. 10.- Surface mass fraction of ${ }^{12} \mathrm{C}$ in terms of the effective temperature for all our white dwarf sequences. Filled circles are the DQ white dwarfs studied by Koester \& Knist (2006). 
It is important to emphasize that the total amount of carbon that is dredged-up to the surface is strongly dependent on the initial chemical profiles, which are a result of the previous evolution of the progenitor stars. Therefore, in order to correctly assess the final surface carbon abundance and to obtain realistic cooling times, it is important to calculate the complete progenitor evolution through the thermally pulsing phase, and more importantly during the born again episode, where the chemical profiles of carbon and oxygen throughout the envelope that will characterize the newly born white dwarf are established. Moreover, chemical and thermal diffusion also influence the carbon mixing process, so they must be considered.

\subsection{Evolutionary times}

The cooling times for some of our sequences are displayed as red lines in Fig. 11. In addition, we also plot in this figure the cooling tracks for hydrogen-rich white dwarfs of

Camisassa et al. (2016), that have similar masses to those considered in this work. The cooling time is defined as zero at the beginning of the white dwarf cooling phase, when the star reaches the maximum effective temperature. The cooling times of all our sequences are also listed in Table 2 for some selected stellar luminosities. Some relevant features of Fig. 11 are worth commenting. In particular, at intermediate luminosities, hydrogen-deficient white dwarfs evolve markedly slower than their hydrogen-rich counterparts. As explained previously in connection with Fig. 7, this is because convective coupling (and the associated release of internal energy) occurs at higher luminosities in hydrogen-deficient white dwarfs, with the consequent lengthening of cooling times at those luminosities. In addition the energy release resulting from crystallization occurs at higher luminosities in hydrogendeficient white dwarfs, thus increasing the cooling times. By contrast, at low-luminosities, hydrogen-deficient white dwarfs evolve faster than hydrogen-rich white dwarfs. This is 
because at those stages the thermal energy content of the hydrogen-deficient white dwarfs is smaller, and also because for these white dwarfs their outer layers are more transparent. In fact, note that hydrogen-deficient white dwarfs evolve to $\log \left(L / L_{\odot}\right) \sim-5$ in less than 8 Gyr, as compared with the time needed by hydrogen-rich white dwarfs to reach the same luminosity, $\sim 14$ Gyr.

We remind the reader that our sequences experience appreciable carbon dredge-up. Carbon enrichment increases the opacity in the non-degenerate outer layers, so energy transport across these layers becomes less efficient. Thus, cooling is delayed and the evolutionary times increase. To estimate the impact of the carbon enrichment of the envelope on the white dwarf evolutionary timescales, we performed additional evolutionary calculations in which convective mixing in the envelope was suppressed, thus forcing our white dwarf envelopes to remain composed by pure helium. Fig. 12 shows the resulting cooling times, together with the cooling times for the sequences with carbon contamination in the envelopes discussed in the preceding paragraph. Note that carbon dredge-up markedly delays the cooling process and increases the evolutionary times. These delays start to manifest themselves at very low surface luminosities. In fact, delays in cooling times of the order of 1 Gyr are reached at $\log \left(L / L_{\odot}\right) \sim-5$.

The observational appearance of our theoretical cooling tracks can be obtained from the radiative transfer calculations performed with the atmospheric numerical code. Fig. 13 presents a color-color diagram in the Sloan Digital Sky Survey (SDSS) photometric system, showing the cooling tracks of hydrogen-deficient white dwarfs with masses $0.51,0.58$, 0.66 and $0.87 M_{\odot}$. Observed white dwarfs with spectroscopically determined pure helium atmospheres (Koester \& Kepler 2015) - that is, of the DB spectral type - are plotted using open circles. Observed white dwarfs with atmospheres composed by helium with traces of carbon (Harris et al. 2003; Kleinman et al. 2004) - that is, of the DQ spectral 


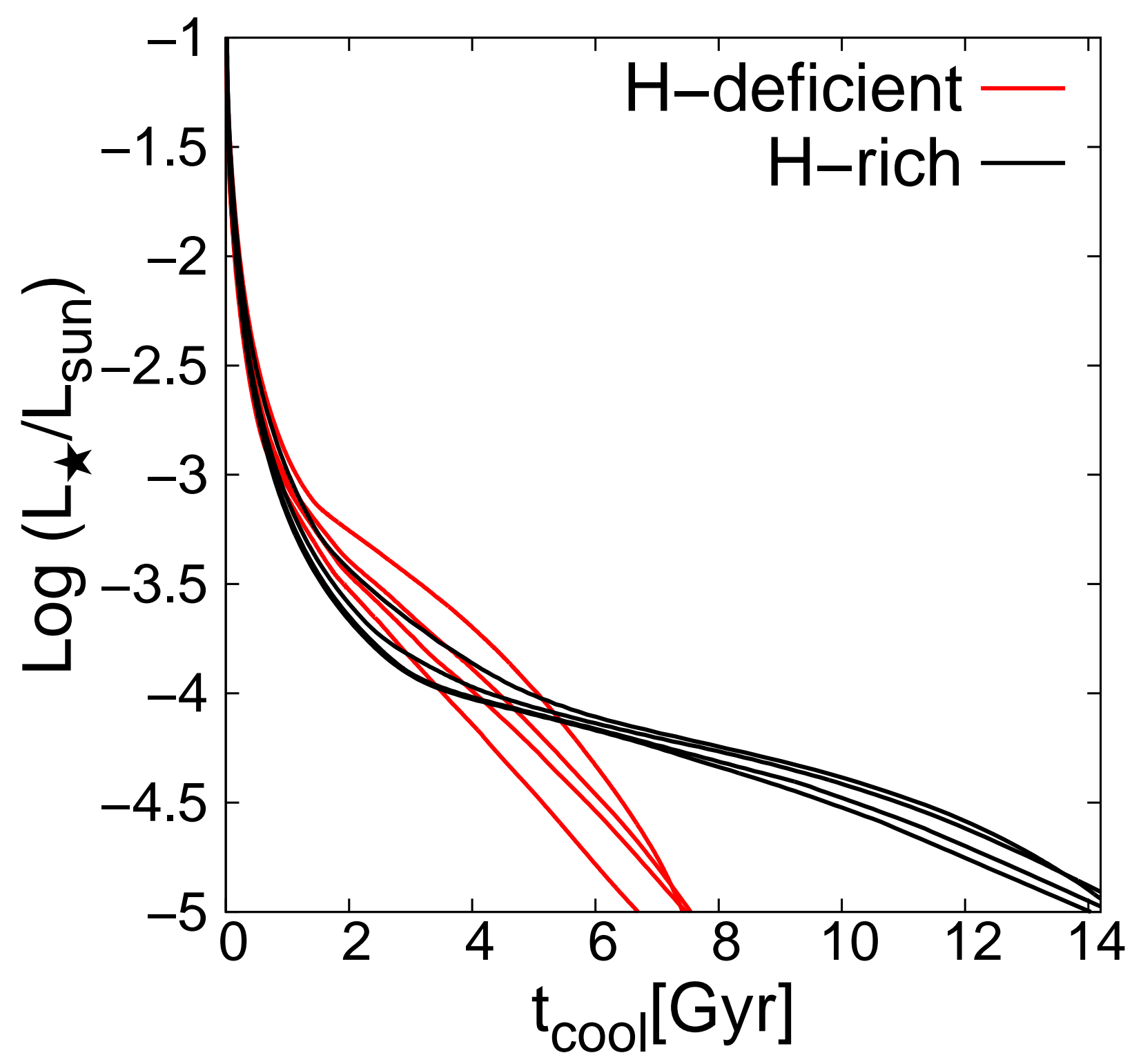

Fig. 11.- White dwarf evolutionary times. The red solid lines indicate four hydrogendeficient white dwarf sequences calculated in this work. At $t_{\mathrm{cool}}=6 \mathrm{Gyr}$ and from bottom to top, the different lines display the $0.51,0.58,0.66$ and $0.87 M_{\odot}$ model sequences, respectively. Black solid lines correspond to hydrogen-rich white dwarf tracks with similar masses from Camisassa et al. (2016). 

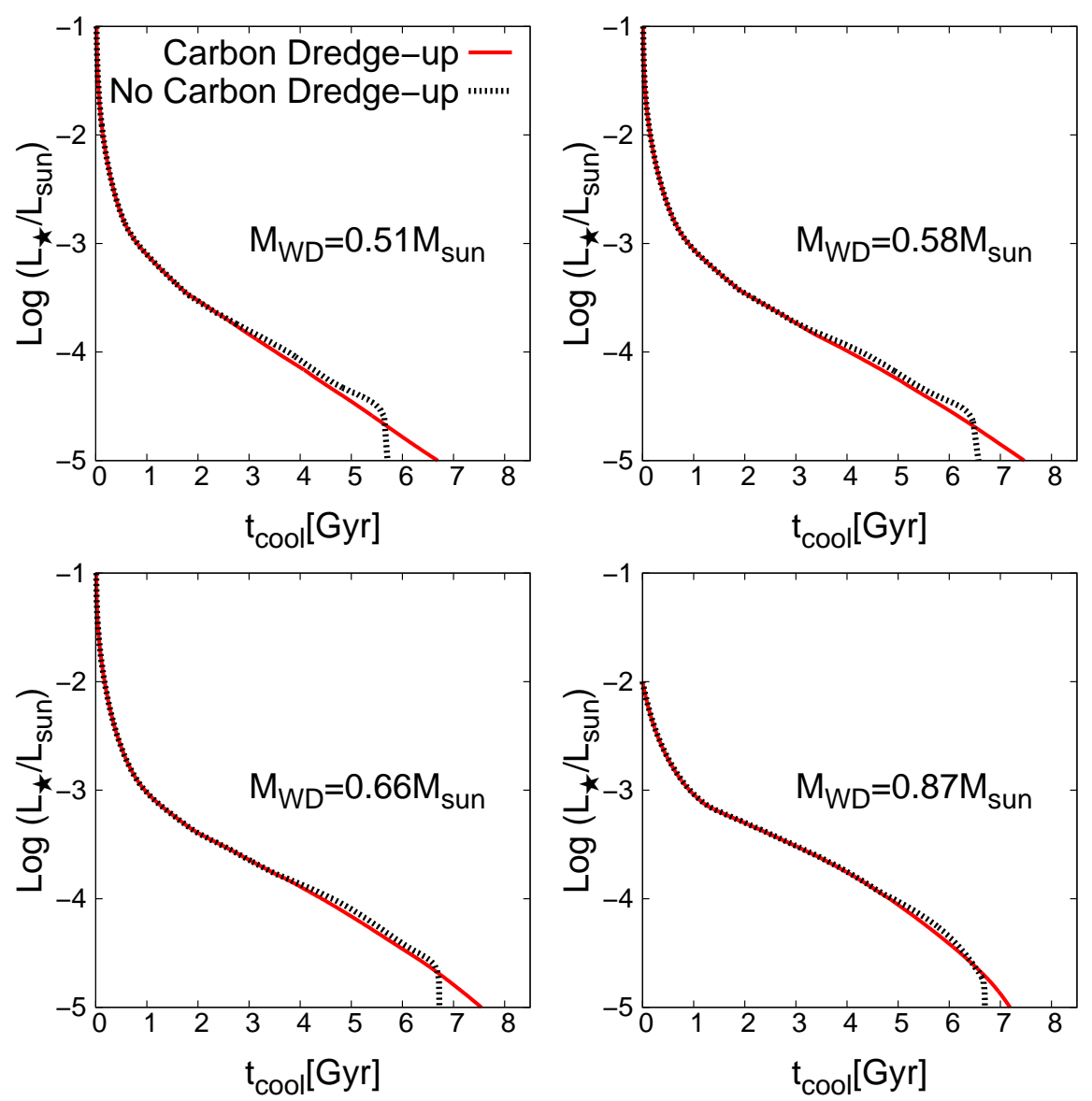

Fig. 12.- White dwarf evolutionary times for the sequences in which carbon is allowed to be dredged-up to the surface by convection (red solid lines), and for the sequences in which convective mixing was suppressed and, therefore, the envelopes are composed of pure helium (black dotted lines). All the sequences have been calculated using our detailed model atmospheres for pure helium composition as outer boundary conditions. 


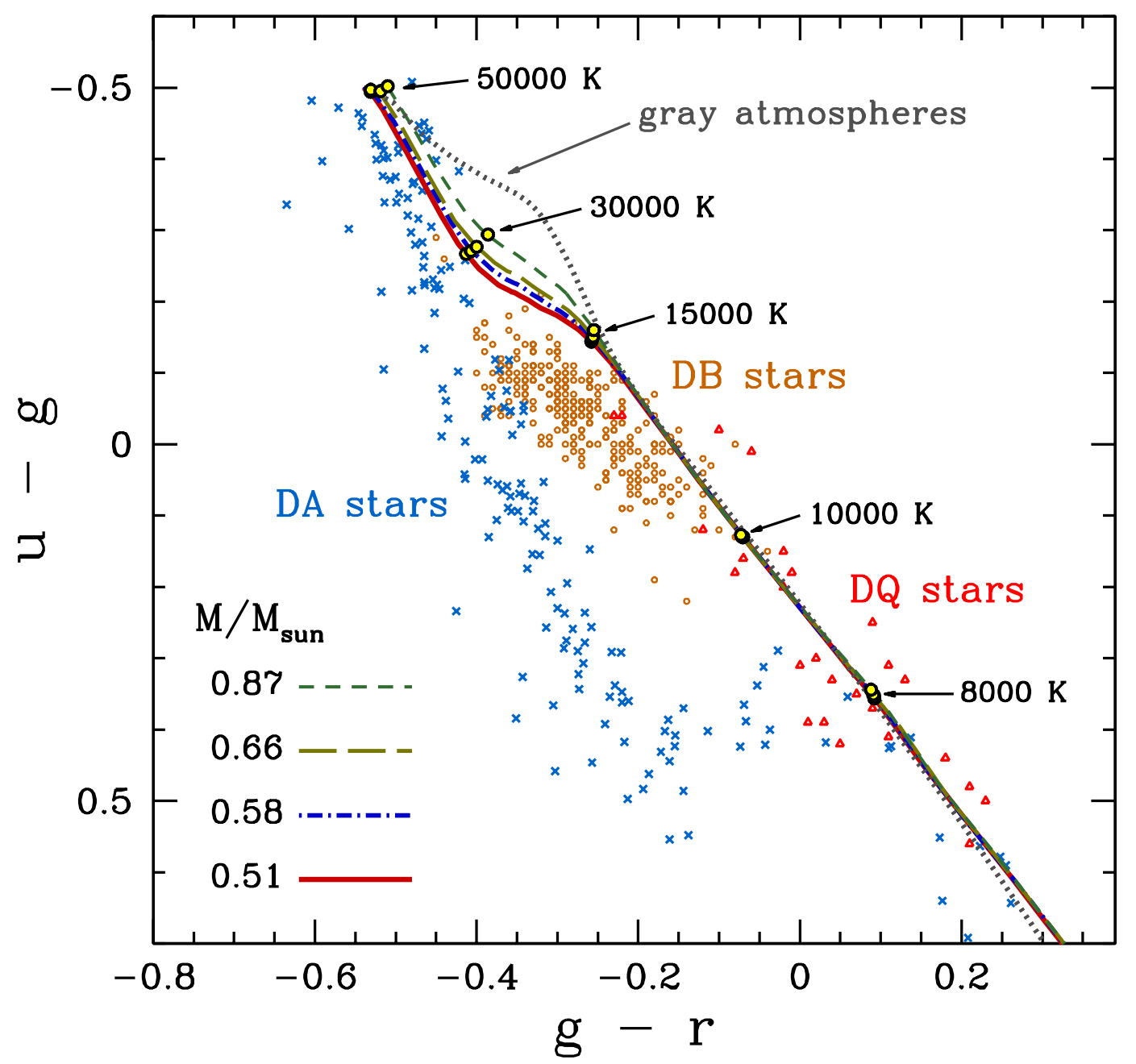

Fig. 13.- $(u-g)$ versus $(g-r)$ color-color diagram in the SDSS photometric system for the $0.51,0.58,0.66$ and $0.87 M_{\odot}$ cooling tracks of hydrogen-deficient white dwarfs. The $0.87 M_{\odot}$ curve is labeled with effective temperatures. The dotted line displays the same evolutionary tracks calculated considering the gray approximation. Open circles are the DB white dwarfs studied by Koester \& Kepler (2015), open triangles are the DQ white dwarfs studied by Harris et al. (2003) and Kleinman et al. (2004), and crosses are the DA white dwarfs studied by Holberg \& Bergeron (2006). 
type - are plotted using open triangles. Those white dwarfs with hydrogen atmospheres (Holberg \& Bergeron 2006) - that is, of the DA spectral type - are represented using crosses. There is a considerable scatter in the observed colors of these stars, with the DB and DQ white dwarfs located in the middle of the diagram, and DA white dwarfs located at the left of the helium sequences. Note that the theoretical colors of our white dwarf models increase steadily along the evolution. The theoretical colors show a moderate dependence on the stellar mass for effective temperatures above $T_{\text {eff }} \approx 15000 \mathrm{~K}$, while all sequences fall on a same line at lower effective temperatures. It should be emphasized that our white dwarf sequences change their spectral type from DB to DQ at $T_{\text {eff }} \approx 15000 \mathrm{~K}$ as a result of carbon dredge-up due to convective mixing. The agreement with the observational data is quite good. Also, for the sake of comparison, we have calculated the observational appearance of the same cooling tracks but considering the gray approximation (dotted line). The effect of solving the radiative transfer for each frequency is obvious in the regions where the helium opacity has a strong dependence on the wavelength, this is for $T_{\text {eff }} \gtrsim 15000 \mathrm{~K}$ and for $T_{\text {eff }} \lesssim 7000 \mathrm{~K}$. We finally stress that our model atmospheres represent a considerable improvement over the gray atmospheres that most models employ.

\section{Summary and conclusions}

We have computed evolutionary cooling sequences for hydrogen-deficient white dwarfs resulting from Solar metallicity progenitors, aimed at providing reliable evolutionary cooling tracks for these stars. For that purpose, in our calculations we considered detailed non-gray model atmospheres made of pure helium. These model atmospheres take into account the most advanced prescriptions of high-density effects on the radiative processes and in the equation of state, as described in Sect. 2.2. Moreover, for our white dwarf evolutionary calculations also we took into account the predictions of full evolutionary calculations of the 
corresponding progenitor stars, as explained in Sect. 2.3. These calculations encompass the full history of the progenitor star, since they start at the ZAMS, and were evolved through the thermally pulsing AGB and, most importantly, through the born-again scenario, which dictates the final chemical composition of the outer layers of our model white dwarfs. Therefore, the initial chemical profiles of our evolving white dwarfs are self-consistent and realistic, as they result from the previous evolution of the progenitors of white dwarfs. Moreover, these chemical profiles were evolved taking into account element diffusion. Finally, the mass interval of our cooling sequences spans from $\sim 0.5 M_{\odot}$ to $\sim 1.0 M_{\odot}$, and thus cover the entire range of masses typical of carbon-oxygen white dwarfs. In short, the set of white dwarf cooling tracks presented here is the first suite of homogeneous evolutionary calculations of hydrogen-deficient white dwarfs in the literature that consider non-gray atmospheres, include in detail the density effects on the energy transport in the cool helium atmosphere, element diffusion, consider the entire evolutionary history of the corresponding progenitor stars, and cover the full range of masses of interest. Consequently, they can be considered as an important step forward in this kind of calculations. 1 .

Our calculations show that convective coupling occurs at larger luminosities in hydrogen-deficient white dwarfs than in hydrogen-rich white dwarfs. As a result of the associated release of internal energy, at intermediate-luminosities hydrogen-deficient white dwarfs evolve slower than hydrogen-rich white dwarfs. At lower luminosities the reverse occurs. In particular, hydrogen-deficient white dwarfs reach luminosities as low as $\log \left(L / L_{\odot}\right) \sim-5$ in less than $8 \mathrm{Gyr}$, whereas hydrogen-rich white dwarfs reach this luminosity in about 14 Gyr, see Sect. 3.2.

It is important to highlight that in all our hydrogen-deficient white dwarf sequences,

\footnotetext{
${ }^{1}$ The evolutionary sequences presented in this work can be downloaded from http://evolgroup.fcaglp.unlp.edu.ar.
} 
at $T_{\text {eff }} \sim 15000 \mathrm{~K}$, the outer convective zone reaches the carbon-rich layers, and the surface carbon abundance increases as a result of convective mixing. The chemical enrichment in carbon of the very outer layers of our model stars has a direct consequence, as the spectral type of our model white dwarfs evolves from DB to DQ. This is consistent with the properties of the observed white dwarf population. We calculated the changes in the chemical abundances due to convective mixing and element diffusion, coupled with the thermal evolution of our white dwarf models. We found that the carbon enrichment is larger when the outer boundary conditions are those predicted by our detailed model atmospheres compared to the case in which Eddington atmospheres are considered. We also found that (quite naturally) the cooling times of hydrogen-deficient white dwarfs depend on the amount of carbon dredged-up to the surface by convection. In particular, carbon dredge-up results in marked delays on the evolutionary times at very low surface luminosities. In this sense, it is worth emphasizing that detailed initial chemical profiles in the envelope of these white dwarfs, as predicted by evolution of their progenitor stars during the last thermal pulse, are crucial. Moreover, our prediction that the outer layers are enriched in carbon, implies that the chemical stratification predicted by evolution of their progenitor stars is in line with the carbon enrichment inferred from observations.

Our results also show that detailed helium model atmospheres are needed to compute reliable cooling times for hydrogen-deficient white dwarfs. Although our cooling sequences represent a clear improvement over previous efforts in computing the evolution of such white dwarfs, a cautionary remark is in order at this point. As mentioned, at low effective temperatures, the pristine helium envelopes of hydrogen-deficient white dwarfs become convective, and their atmospheres are contaminated by the dredged-up material. This has a consequence that our models do not consider. Indeed, carbon contamination in the atmosphere, of course, results in an additional source of opacity, and consequently the atmospheres of white dwarfs made of almost pure helium become more opaque. However, 
to the best of our knowledge, calculations of the carbon opacity for the thermodynamic conditions prevailing in such dense atmospheres do not exist yet. Consequently, in this first set of calculations we did not attempt to incorporate the effects of the enhanced carbon opacity on the model atmospheres. Hence, our evolutionary cooling times at very low effective temperatures should be taken with some caution. However, as mentioned, for larger effective temperatures our calculations are clearly superior to those existing now. Thus, we defer the calculation of improved cooling sequences at very low effective temperatures taking into account the effects of metal contamination — to forthcoming works.

We warmly thank M. M. Miller Bertolami for a useful discussion that helped to improved the final version of the paper. Part of this work was supported by AGENCIA through the Programa de Modernización Tecnológica BID 1728/OC-AR, by the PIP 112-200801-00940 and PIP 112-200801-01474 grant from CONICET, by MINECO grant AYA2014-59084-P, and by the AGAUR. This research has made use of NASA Astrophysics Data System. 
Table 2: Cooling times of our hydrogen-deficient white dwarf models at selected luminosities.

\begin{tabular}{cccccccc}
\hline \hline $\log \left(L / L_{\odot}\right)$ & \multicolumn{7}{c}{$t(\mathrm{Gyr})$} \\
\hline \multirow{2}{*}{2.0} & $0.51\left(M_{\odot}\right)$ & $0.54 M_{\odot}$ & $0.58 M_{\odot}$ & $0.66 M_{\odot}$ & $0.74 M_{\odot}$ & $0.87 M_{\odot}$ & $1.00 M_{\odot}$ \\
\cline { 2 - 8 } 3.0 & 0.142 & 0.136 & 0.151 & 0.173 & 0.193 & 0.224 & 0.259 \\
3.5 & 0.813 & 0.882 & 0.909 & 0.969 & 1.030 & 1.148 & 1.346 \\
4.0 & 1.911 & 2.006 & 2.161 & 2.425 & 2.738 & 3.165 & 3.314 \\
4.5 & 3.527 & 3.864 & 4.056 & 4.429 & 4.792 & 5.055 & 4.901 \\
5.0 & 5.136 & 5.790 & 5.890 & 6.129 & 6.399 & 6.438 & 5.913 \\
\hline \hline
\end{tabular}




\section{REFERENCES}

Althaus, L. G., Camisassa, M. E., Miller Bertolami, M. M., Córsico, A. H., \& García-Berro, E. 2015, A\&A, 576, A9

Althaus, L. G., Córsico, A. H., Bischoff-Kim, A., et al. 2010a, ApJ, 717, 897

Althaus, L. G., Córsico, A. H., Isern, J., \& García-Berro, E. 2010b, A\&A Rev., 18, 471

Althaus, L. G., García-Berro, E., Isern, J., Córsico, A. H., \& Miller Bertolami, M. M. 2012, A\&A, 537, A33

Althaus, L. G., Miller Bertolami, M. M., \& Córsico, A. H. 2013, A\&A, 557, A19

Althaus, L. G., Panei, J. A., Miller Bertolami, M. M., et al. 2009, ApJ, 704, 1605

Althaus, L. G., Serenelli, A. M., Córsico, A. H., \& Montgomery, M. H. 2003, A\&A, 404, 593

Althaus, L. G., Serenelli, A. M., Panei, J. A., et al. 2005, A\&A, 435, 631

Becker, A., Lorenzen, W., Fortney, J. J., et al. 2014, ApJS, 215, 21

Benvenuto, O. G., \& Althaus, L. G. 1999, MNRAS, 303, 30

Berengut, J. C., Flambaum, V. V., Ong, A., et al. 2013, Physical Review Letters, 111, 010801

Bergeron, P., Wesemael, F., \& Beauchamp, A. 1995, PASP, 107, 1047

Bischoff-Kim, A., Montgomery, M. H., \& Winget, D. E. 2008, ApJ, 675, 1512

Bono, G., Salaris, M., \& Gilmozzi, R. 2013, A\&A, 549, A102

Camisassa, M. E., Althaus, L. G., Córsico, A. H., et al. 2016, ApJ, 823, 158 
Cassisi, S., Potekhin, A. Y., Pietrinferni, A., Catelan, M., \& Salaris, M. 2007, ApJ, 661, 1094

Córsico, A. H., Althaus, L. G., Miller Bertolami, M. M., et al. 2012, MNRAS, 424, 2792

Farihi, J. 2016, New A Rev., 71, 9

Ferguson, J. W., Alexander, D. R., Allard, F., et al. 2005, ApJ, 623, 585

Fontaine, G., \& Brassard, P. 2008, PASP, 120, 1043

Fontaine, G., Brassard, P., \& Bergeron, P. 2001, PASP, 113, 409

García-Berro, E., Hernanz, M., Isern, J., \& Mochkovitch, R. 1988, Nature, 333, 642

-. 1995, MNRAS, 277, 801

Garcia-Berro, E., Hernanz, M., Mochkovitch, R., \& Isern, J. 1988, A\&A, 193, 141

García-Berro, E., Lorén-Aguilar, P., Torres, S., Althaus, L. G., \& Isern, J. 2011, J. Cosmology Astropart. Phys., 5, 21

García-Berro, E., \& Oswalt, T. D. 2016, New A Rev., 72, 1

García-Berro, E., Torres, S., Isern, J., \& Burkert, A. 1999, MNRAS, 302, 173

-. 2004, A\&A, 418, 53

García-Berro, E., Torres, S., Althaus, L. G., et al. 2010, Nature, 465, 194

Gustafsson, B., \& Nissen, P. E. 1972, A\&A, 19, 261

Haft, M., Raffelt, G., \& Weiss, A. 1994, ApJ, 425, 222

Hansen, B. M. S. 1998, Nature, 394, 860 
-. 1999, ApJ, 520, 680

Hansen, B. M. S., Kalirai, J. S., Anderson, J., et al. 2013, Nature, 500, 51

Harris, H. C., Liebert, J., Kleinman, S. J., et al. 2003, AJ, 126, 1023

Holberg, J. B., \& Bergeron, P. 2006, AJ, 132, 1221

Horowitz, C. J., Schneider, A. S., \& Berry, D. K. 2010, Physical Review Letters, 104, 231101

Hummer, D. G., \& Mihalas, D. 1988, ApJ, 331, 794

Iglesias, C. A., \& Rogers, F. J. 1996, ApJ, 464, 943

Iglesias, C. A., Rogers, F. J., \& Saumon, D. 2002, ApJ, 569, L111

Isern, J., García-Berro, E., Althaus, L. G., \& Córsico, A. H. 2010, A\&A, 512, A86

Isern, J., García-Berro, E., Hernanz, M., Mochkovitch, R., \& Torres, S. 1998, ApJ, 503, 239

Isern, J., García-Berro, E., Torres, S., \& Catalán, S. 2008, ApJ, 682, L109

Isern, J., Hernanz, M., \& García-Berro, E. 1992, ApJ, 392, L23

Isern, J., Hernanz, M., Mochkovitch, R., \& Garcia-Berro, E. 1991, A\&A, 241, L29

Itoh, N., Hayashi, H., Nishikawa, A., \& Kohyama, Y. 1996, ApJS, 102, 411

Ivezic, Z., Axelrod, T., Brandt, W. N., et al. 2008, Serbian Astronomical Journal, 176, 1

Jeffery, E. J., von Hippel, T., DeGennaro, S., et al. 2011, ApJ, 730, 35

Kleinman, S. J., Harris, H. C., Eisenstein, D. J., et al. 2004, ApJ, 607, 426

Koester, D., \& Kepler, S. O. 2015, A\&A, 583, A86 
Koester, D., \& Knist, S. 2006, A\&A, 454, 951

Kowalski, P. M. 2014, A\&A, 566, L8

Magni, G., \& Mazzitelli, I. 1979, A\&A, 72, 134

Miller Bertolami, M. M. 2016, A\&A, 588, A25

Miller Bertolami, M. M., \& Althaus, L. G. 2006, A\&A, 454, 845

Miller Bertolami, M. M., Althaus, L. G., Unglaub, K., \& Weiss, A. 2008, A\&A, 491, 253

Miller Bertolami, M. M., Rohrmann, R. D., Granada, A., \& Althaus, L. G. 2011, ApJ, 743, L33

Napiwotzki, R., Christlieb, N., Drechsel, H., et al. 2001, Astronomische Nachrichten, 322, 411

Renedo, I., Althaus, L. G., Miller Bertolami, M. M., et al. 2010, ApJ, 717, 183

Rohrmann, R. D., Althaus, L. G., García-Berro, E., Córsico, A. H., \& Miller Bertolami, M. M. 2012, A\&A, 546, A119

Rohrmann, R. D., Serenelli, A. M., Althaus, L. G., \& Benvenuto, O. G. 2002, MNRAS, 335,499

Salaris, M., Althaus, L. G., \& García-Berro, E. 2013, A\&A, 555, A96

Salaris, M., Cassisi, S., Pietrinferni, A., Kowalski, P. M., \& Isern, J. 2010, ApJ, 716, 1241

Salaris, M., Domínguez, I., García-Berro, E., et al. 1997, ApJ, 486, 413

Salaris, M., García-Berro, E., Hernanz, M., Isern, J., \& Saumon, D. 2000, ApJ, 544, 1036 
Santamaría-Pérez, D., Mukherjee, G. D., Schwager, B., \& Boehler, R. 2010, Phys. Rev. B, 81,214101

Segretain, L., Chabrier, G., Hernanz, M., et al. 1994, ApJ, 434, 641

Tassoul, M., Fontaine, G., \& Winget, D. E. 1990, ApJS, 72, 335

Torres, S., García-Berro, E., Althaus, L. G., \& Camisassa, M. E. 2015, A\&A, 581, A90

Torres, S., García-Berro, E., Burkert, A., \& Isern, J. 2002, MNRAS, 336, 971

Torres, S., García-Berro, E., Isern, J., \& Figueras, F. 2005, MNRAS, 360, 1381

Wachlin, F. C., Miller Bertolami, M. M., \& Althaus, L. G. 2011, A\&A, 533, A139

Weiss, A., \& Ferguson, J. W. 2009, A\&A, 508, 1343

Winget, D. E., Hansen, C. J., Liebert, J., et al. 1987, ApJ, 315, L77

Winget, D. E., \& Kepler, S. O. 2008, ARA\&A, 46, 157

Wood, M. A. 1992, ApJ, 386, 539

Wood, M. A. 1995, in Lecture Notes in Physics, Berlin Springer Verlag, Vol. 443, White Dwarfs, ed. D. Koester \& K. Werner, 41

York, D. G., Adelman, J., Anderson, Jr., J. E., et al. 2000, AJ, 120, 1579 